\title{
Author Correction to: Active Control of Offshore Steel Jacket Platforms
}

\section{Author Correction to:}

B.-L. Zhang et al., Active Control of Offshore Steel Jacket Platforms, https://doi.org/10.1007/978-981-13-2986-9

The book was inadvertently published with an incorrect Grant number. In Front Matter, 4th line of page number vii, the sentence "of China under Grants Z19F030002,..." has been replaced by "of China under Grants LZ19F030001,...”. 